\title{
A Comparison of Image Processing Techniques for Optical Interference Fringe Analysis
}

\author{
Muhammad FAROOQ ${ }^{1}$, Aasma ASLAM ${ }^{2}$, Babar HUSSAIN ${ }^{1,3^{*}}$, \\ Ghazanfar HUSSAIN ${ }^{1}$, and Masroor IKRAM ${ }^{4}$
}

\author{
${ }^{1}$ National Institute of Lasers and Optronics, Lehtrar Road, Islamabad, 45650, Pakistan \\ ${ }^{2}$ Hazara University, Mansehra and NUST, Islamabad, 21300, Pakistan \\ ${ }^{3}$ University of North Carolina at Charlotte, Department of Electrical and Computer Engineering, 9201 University City \\ Boulevard, Charlotte, 28223, USA \\ ${ }^{4}$ Pakistan Institute of Engineering and Applied Sciences, Islamabad, 45650, Pakistan \\ "Corresponding author: Babar HUSSAIN_E-mail: bhussai1@uncc.edu
}

\begin{abstract}
We present a comparison of different techniques for the analysis of the shift and tilt in optical interference fringes. Fringe center, Radon transform, and Gaussian approximation methods are used for fringe analysis. We have measured the tilt and shift between two relevant fringe patterns. The error in tilt measurement was about $2 \%$, and the displacement of the order of few nanometers was measured by the fringe shift analysis. The comparison between the techniques is analyzed with respect to percentage error.
\end{abstract}

Keywords: Interferometry, fringes, tilt, shift, imaging

Citation: Muhammad FAROOQ, Aasma ASLAM, Babar HUSSAIN, Ghazanfar HUSSAIN, and Masroor IKRAM, "A Comparison of Image Processing Techniques for Optical Interference Fringe Analysis," Photonic Sensors, 2015, 5(4): 304-311.

\section{Introduction}

Interferometry is one of the most established techniques of measurement that dates back to Michelson-Morley experiment [1]. Applications of optical interferometry include interference lithography for the fabrication of large area variable period gratings [2], surface metrology [3], vibration analysis [4-6], astronomical optical interferometry e.g. space interferometry mission, 2009 [7] etc. Thickness and refractive index of thin films can also be measured by interferometry with higher accuracy than that in other optical techniques [8, 9]. The important part in all the interferometric setups is the analysis of fringe data which is mainly done through fringe analysis software. Various techniques are reported in literature for fringe analysis that may be classified as three main categories [10]:

(1) Intensity based analysis

(2) Transformation based analysis

(3) Phase based analysis

Intensity based techniques which are directly applied to greyscale images are faster but more prone/subtle to noise. In transformation based approach, interferograms are transformed to perform analysis in some other domains e.g. Fourier transform [11] and tavelet transform [12]. In phase based method, the wrapped phase of interferograms is extracted and analyzed [13].

Previously, we have reported two electronic

Received: 30 March 2015 / Revised: 16 July 2015

(C) The Author(s) 2015. This article is published with open access at Springerlink.com

DOI: $10.1007 / \mathrm{s} 13320-015-0254-\mathrm{z}$

Article type: Regular 
processing techniques for fringe analysis to be used in displacement/vibration sensors $[5,6]$. In this paper, we report a comparison of different image processing techniques for interference based fringe analysis. We have measured the displacement and tilt of an object. For the measurement of the fringe displacement, we have used the fringe center method (FCM) [14-16] and Gaussian approximation method (GAM). Meanwhile, for the measurement of the tilt, we have used FCM and Radon transform method (RTM) [17, 18]. Previously reported error in rotation is on the order of one degree. We have used image detection resolutions of $180 \times 180$ and $1000 \times 1000$. We have employed additional preprocessing steps such as contrast enhancement, image filtering, and morphological treatment. We have achieved a precision of one arc minute with RTM which is the best in all the above mentioned techniques.

\section{Experimental setup}

We used a Michelson interferometer with one of its mirrors slightly tilted to achieve linear fringes and used a He-Ne laser at the green wavelength $543 \mathrm{~nm}$ as a light source; the setup is shown in Fig. 1. The mirror could be rotated around two axes to tilt the fringes at any angle within $360^{\circ}$. The tilted fringes were recorded by a charge coupled device (CCD) camera, and the image processing techniques were applied to find the tilt. The same setup was used for translation of fringes by translating

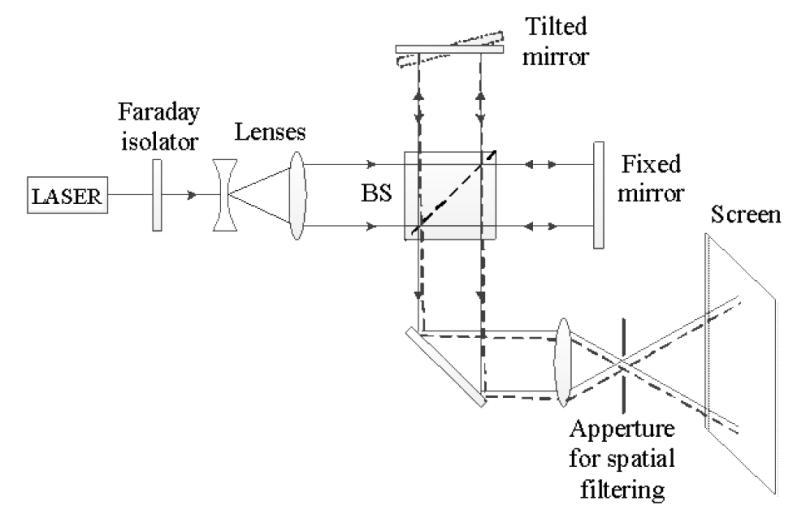

Fig. 1 Michelson interferometer based experimental setup ( BS: beamsplitter). one of the mirrors along the laser beam. The rotating mirror had two micrometers attached with it to produce the desired tilt.

The recorded interferogram is shown in Fig.2(a). The actual mirror tilt angle is a function of two axial motions. Figure 2(b) shows this scheme, and Fig. 2(c) further explains the tilt angle geometry. The distances $C X$ and $C Y$ were $85 \mathrm{~mm}$ (confirmed by Newport Company). Angles $\theta_{x}$ and $\theta_{y}$ were found by simple trigonometry using known micrometer displacements $m_{1}$ and $m_{2}$, respectively. Figure 2(d) shows the mount used for the mirror to be tilted.

$$
\begin{gathered}
\theta_{x}=\tan ^{-1}\left(\frac{m_{1}}{C X}\right) \\
\theta_{y}=\tan ^{-1}\left(\frac{m_{2}}{C X}\right) \\
d x=r \tan \theta_{x} \\
d y=r \tan \theta_{y} \\
\psi=\tan ^{-1}\left(\frac{d y}{d x}\right) .
\end{gathered}
$$

The final result can be written as

$$
\psi=\tan ^{-1}\left(\frac{m_{2} / C Y}{m_{1} / C X}\right) .
$$

(a)

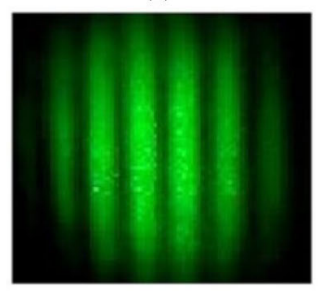

(c)

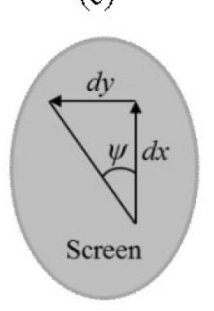

(b)

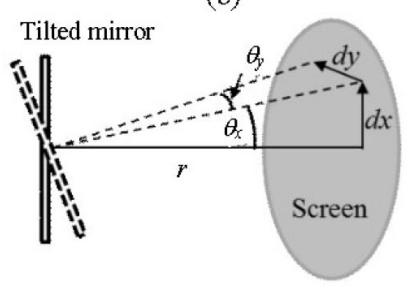

(d)

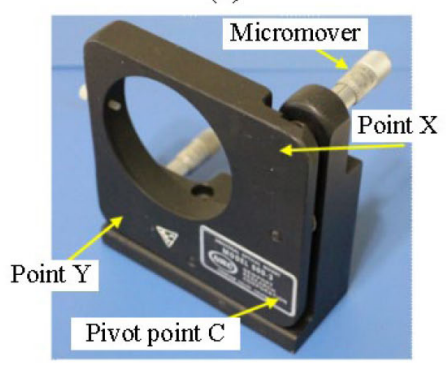

Fig. 2 Mathematical modeling of the fringes tilt: (a) recorded fringes image, (b) tilt mechanism using two micrometers at points $\mathrm{X}$ and $\mathrm{Y}$, (c) geometry of the tilt scheme showing the final rotation $\psi$ in the fringes, and (d) mechanical setup for the manual tilt in the tilted mirror around two axes of rotation (X and $\mathrm{Y}$ : position points of the two micrometers; $\mathrm{C}$ : the pivot point). 


\section{Interference fringes tilt analysis}

\subsection{Fringe center method}

In the fringe center method, we found the centerlines of the interference fringes which actually correspond to the fringe maxima [14-16]. After "binarization", we chose the top left and top right, bottom left and bottom right points of the truncated rectangular fringes and then took their mean to find two points on the fringe centerline. Orientation of the individual centerline was found by using these two points on the centerlines. The central point of the fringe (vertically) could be found using the same method.

The choice of morphological operations depends upon the quality of the images as the images may be degraded during thresholding if proper threshold values are not used or the recorded images may be highly affected by environmental disturbances [19]. We used black chart paper as a screen, as it absorbs undesired reflections of light and reduces the undesirable vignetting effect of cameras [20]. Environmental vibrations were controlled using dampers.

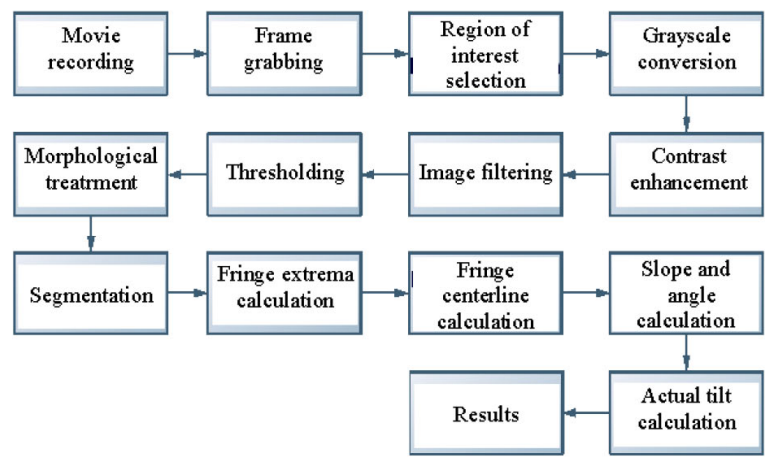

Fig. 3 Sequence of steps followed in the FCM for tilt measurement: contrast enhancement, image filtering, and morphological treatment improve results.

The detailed procedure is charted in Fig. 3. We used adaptive histogram equalization for contrast enhancement and adaptive Wiener filtering to remove noise effects. The Wiener filter is the most suitable choice for this type of application because we may come across small vibrations and blurriness due to unfocused optics. Otsu's thresholding algorithm [20] was used for binarization. Cleaning, majority filling, hole filling, and dilation morphological operations were used to improve images.

\subsection{Radon transform method}

The Radon transform was used in interference fringe analysis software (IFAS) for the measurement of fringe tilts [17]. The Radon transform is a better approach than FCM or Gaussian approximation, and it provides a higher accuracy even for very small tilts. It treats the image as a global object and converts the two dimensional image objects into one dimensional Radon projection [18]. The inverse Radon transform can, of course, be used for reconstruction of the original objects. The Radon transform of a two dimensional function $f(x, y)$, denoted by $R(s, \theta)\{f(x, y)\}$ is defined as

$$
R(s, \theta)\{f\}=\iint_{-\infty} f(x, y) \delta(s-x \cos \theta-y \sin \theta) d x d y
$$

where $s$ is the perpendicular distance of the projection line from the origin of the coordinate axis, and $\theta$ is the angle formed by the distance vector, as shown in Fig. 4. The origin of the coordinate axis is taken to be the central pixel of the image.

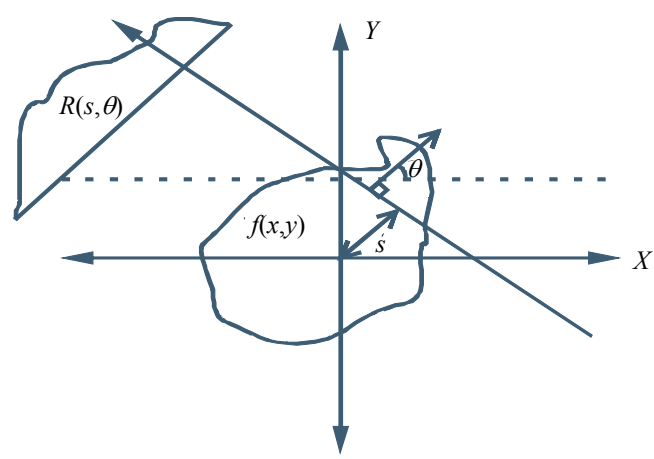

Fig. 4 Schematic showing Radon transform concept.

Basically, the Radon transform finds the orientation of the principal direction of the fringes. We found more than one Radon projection at different angles. All the Radon projections were stored in a matrix whose columns contained Radon 
projections, corresponding to some specific angles. The angle, which corresponded to the absolute maxima of the Radon projection matrix, was the orientation of the principle axis of the texture. The procedure of tilt analysis using the Radon transform is illustrated in Fig. 5.

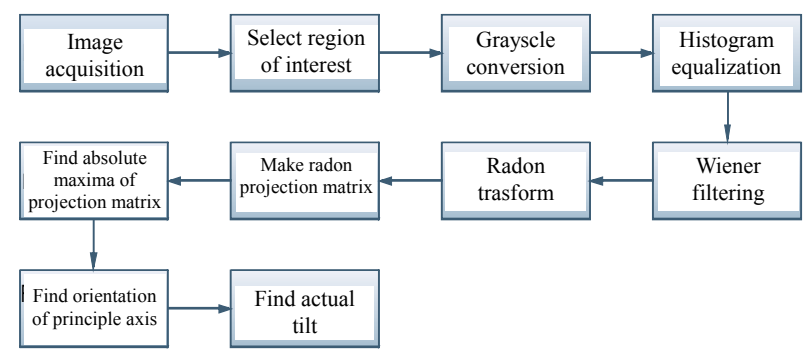

Fig. 5 Radon transform procedure for tilt analysis.

\subsection{Tilt analysis results and discussion}

Table 1 compares two methods based on the experimental results of the tilt analysis using our interference fringe analysis software (IFAS) developed in MATLAB. It can be noticed that the FCM approach gives the worst results for smaller tilts, and the error decreases as the tilt-angle increases. For the better results using the FCM, one needs to increase the resolution. The results have been calibrated using computer generated tilts [e.g. by MATLAB command "imrotate()"], and experimentally, they were given by the tilt scheme explained earlier in the experimental setup. We can conclude that the Radon transform is more accurate than the FCM, even for small tilt measurement.

Table 1 Comparison of FCM and RTM for tilt analysis.

\begin{tabular}{cccccc}
\hline Exp. \# & Input tilt (deg) & $\begin{array}{c}\text { Tilt using } \\
\text { FCM (deg) }\end{array}$ & $\begin{array}{c}\text { Tilt using RTM } \\
(\mathrm{deg})\end{array}$ & $\begin{array}{c}\text { FCM } \\
\text { error } \\
(\%)\end{array}$ & $\begin{array}{c}\text { RTM } \\
\text { error } \\
(\%)\end{array}$ \\
\hline 1 & 0.01667 & 0.00000 & 0.01632 & 100 & 2.05 \\
2 & 0.50000 & 0.36762 & 0.51177 & 26.47 & 2.36 \\
3 & 1.00000 & 1.20941 & 0.98422 & 20.94 & 1.58 \\
4 & 1.50000 & 1.62322 & 1.48846 & 8.21 & 0.77 \\
5 & 2.00000 & 1.94371 & 2.01799 & 2.82 & 0.89 \\
6 & 2.50000 & 2.35891 & 2.48988 & 5.64 & 0.40 \\
7 & 3.00000 & 2.73172 & 3.00274 & 8.94 & 0.09 \\
8 & 4.00000 & 4.08631 & 3.99057 & 2.16 & 0.24 \\
\hline
\end{tabular}

The comparison of error profiles of the two methods for the tilt analysis is shown in the graph of Fig. 6. The graph shows that the error is less in the Radon transform method as compared to the fringe center method. For the FCM, the percent error reduces as the value of tilt increases.

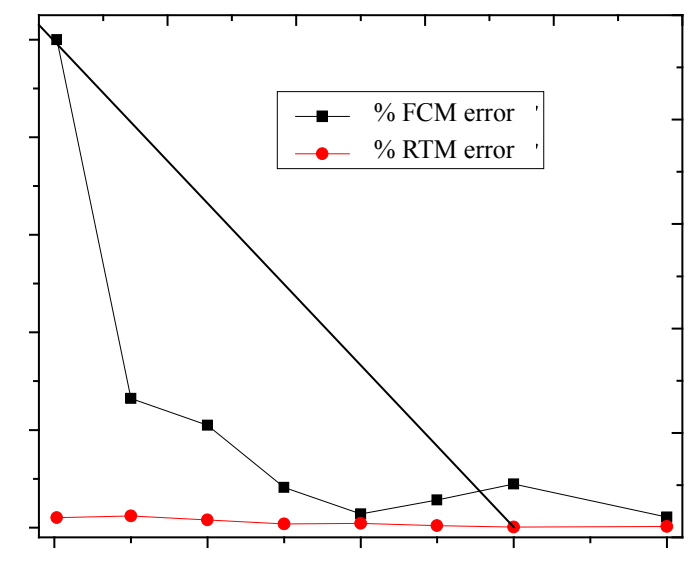

Fig. 6 Comparison of error profiles between the FCM and RTM for the fringe tilt analysis.

\section{Interference fringes shift analysis}

If we introduce some known vibrations to the mirror by using the piezoelectric transducer or by some other mechanisms, the fringes are displaced or shifted. Calculation of this shift is needed in applications such as vibrations analysis, deformation analysis or seismic effects analysis. The interference fringe analysis software implements this feature by two methods i.e. FCM and GAM. The following discussion shows that the FCM is more accurate in this case.

\subsection{Fringe center method}

The complete FCM procedure for shift calculations is shown in Fig. 7. The procedure followed for the fringe shift analysis using the FCM is similar to that of the procedure for the tilt analysis, except that the fringes are first aligned vertically [4] and the least square fitting is used on thinned fringe centerlines. The fringe centerlines have distortions due to roughness of fringe borders. For $180 \times 180$ 
image resolution, the induced error is 0.83393 pixels while the error for $1000 \times 1000$ image resolution is 0.7652 pixels. Ideally, the distance between all consecutive centerlines must be the same, but due to disturbances (e.g. vibrations, camera artifacts, and non-uniform illumination); this distance is not the same for all fringes and is a source of error. To reduce this error, averaging of the consecutive fringe displacement is used. The fringe centerlines plotted on grayscale enhanced and filtered image are shown in Fig. 8.

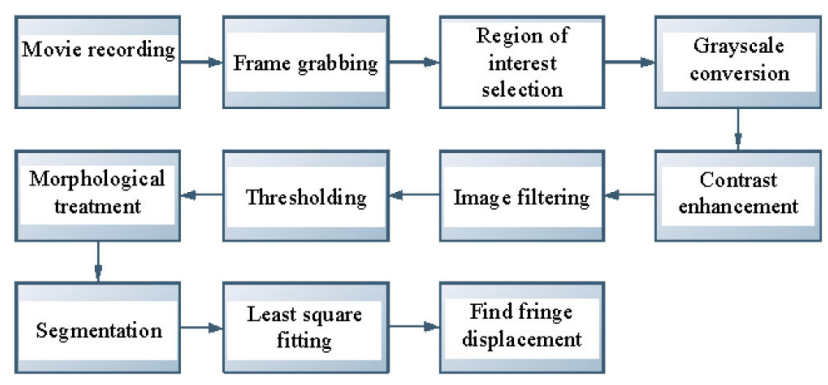

Fig. 7 FCM procedure for shift calculations.

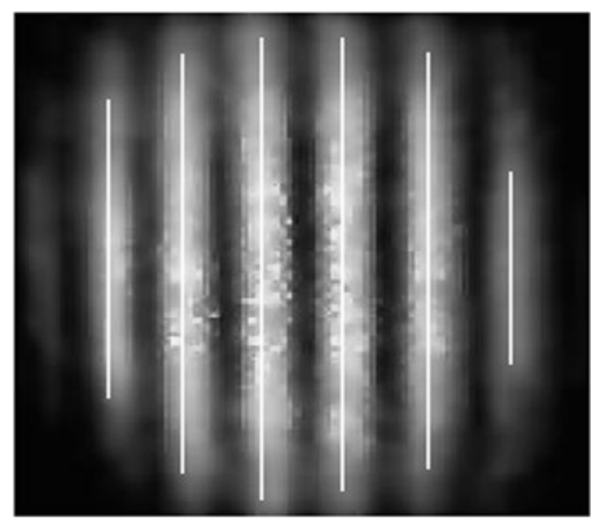

Fig. 8 Interference fringes with center-lines.

\subsection{Gaussian approximation method}

The fringe shift analysis can directly be performed on the intensity images or grayscale image of interferograms [21]. Since we know that the profile of the interferograms is Gaussian, by using this inherent feature of the interferograms, we can find the shift in the interferograms. The method is faster but there are other problems associated with it. Actually, playing with the intensities of the interferograms, yields more error than the binary images using the FCM. This is because of the fact that the intensities of same pixels in two immediate frame images may be different due to image sensors' defects like Vignetting or other environmental disturbances like non-uniform illumination. Also the approximation increases the error, and the error is accumulated. Consequently, the accuracy decreases to a great extent. The procedure, as shown in Fig. 9, starts from movie recording, and we slice two consecutive frames out of it for further processing.

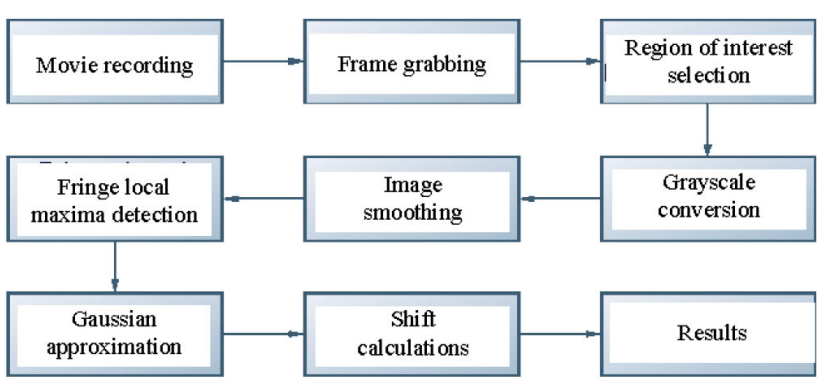

Fig. 9 Procedure for the Gaussian approximation method.

The intensity spectra of the reference image along with approximated Gaussian are shown in Fig. 10. The rising fringe peaks above the Gaussian are because of contrast enhancement. The shifted Gaussians are shown in Fig. 11. Here, one can see that the peaks in the Gaussian profile have been shifted, and we can easily find this displacement in our program.

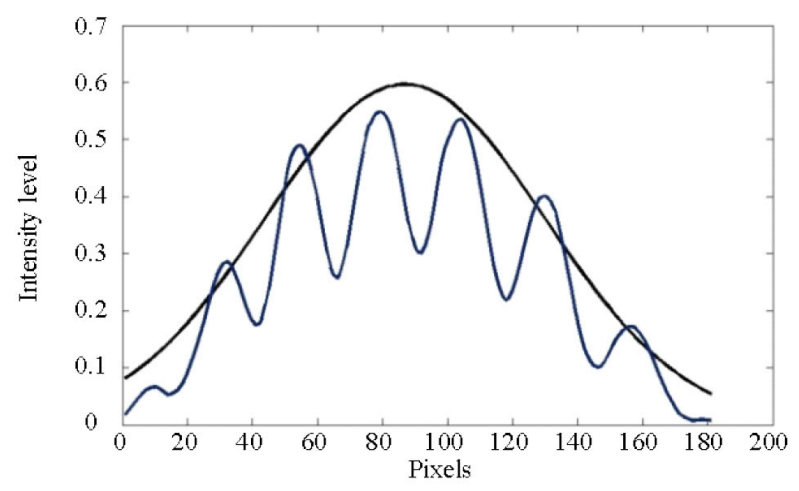

Fig. 10 Reference image intensity spectrum with Gaussian approximation. 


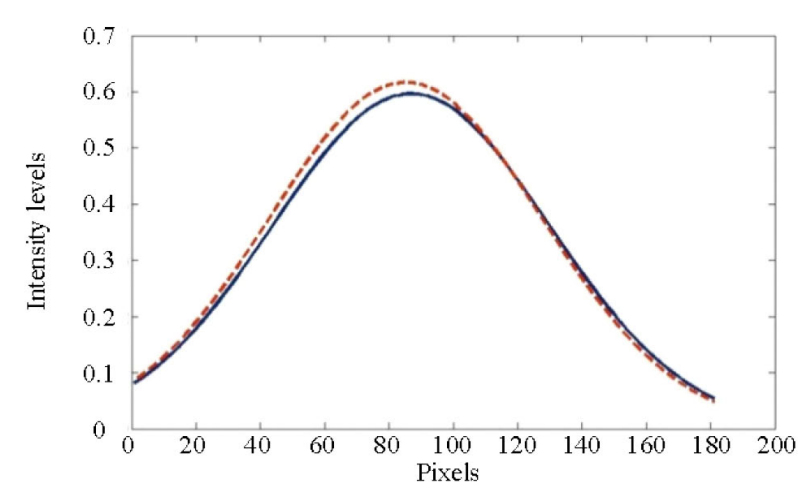

Fig. 11 Shift in Gaussian profile of fringes.

\subsection{Shift analysis results and discussion}

It was analyzed that image resolution plays a key role in the fringe analysis using the FCM. So the experiments were performed on two sets of resolutions i.e. $180 \times 180$ and $1000 \times 1000$. It was concluded that increasing the resolution also increases the precision (or least count) of measurements. From basics of interferometry, the corresponding mirror displacement can be calculated for a complete fringe displacement. So if we can find the total fringe separation in terms of the fact that pixels and wavelength are known, then one can easily find the actual displacement. A precision of $2 \mathrm{~nm}$ shift has been achieved using an image resolution of $1000 \times 1000$. The results are depicted in Table2.

Table 2 Dependence of least count on image resolution.

\begin{tabular}{ccc}
\hline Resolution & Fringe center separation & Least count achieved \\
\hline $180 \times 180$ & 26 pixels & $10.44 \mathrm{~nm}$ \\
$1000 \times 1000$ & 135 pixels & $2.01 \mathrm{~nm}$ \\
\hline
\end{tabular}

For the Gaussian method, the experiment was performed on images of resolution $180 \times 180$ only. It was concluded that the precision of measurements using this method was less than that of the FCM method. If we find the shift in corresponding local maxima, then the error is less than that in finding shift in the Gaussian maxima. The results for a very small shift in fringes are listed in Table 3. The percentage error significantly reduces $(<1 \%)$ for large shifts in fringes.
Table 3 Comparison between the FCM and GAM for the shift analysis.

\begin{tabular}{cc}
\hline Actual shift in fringes & 6 pixels \\
\hline Calculated using FCM & 5 pixels \\
Percentage error using FCM & $16.67 \%$ \\
Calculated using GAM & 3 pixels \\
Percentage error using GAM & $50 \%$ \\
\hline
\end{tabular}

The study presented here can be useful for the optical interference fringe shift and tilt analysis to be employed in interference lithography, surface metrology, mechanical displacement/vibration measurement, astronomical interferometry, study on nonlinear optical materials, optical sensing, and several other applications [22-26].

\section{Conclusions}

Based on the work done, we can conclude that:

(1) The choice of the suitable fringe analysis technique depends upon the application.

(2) For the fringe shift analysis, we suggest that the FCM gives us the better results than that of greyscale intensity image processing methods e.g., Gaussian approximations or by using some window masks such as the fringe peak detection method. The main drawback of the FCM method is its resolution dependence. For the high precision, we need to increase the resolution, but it also increases the computation time as well. So we need some trade-off between these two parameters. Also we need to use extra morphological operations overhead if the images are noisy.

(3) For the fringe tilt analysis, we suggest that the Radon transformation based method is the best solution as it gives very high precision and accuracy. Also it is more efficient than the FCM, PCA, hough transforms or other kinds of transformation based approaches. For tilt applications, we found that the FCM is not an efficient method for finding tilts of the order of arc minutes and arc seconds. We found problems in finding the exact centerline in small tilts, and the error increases even more for smaller tilts. 
Open Access This article is distributed under the terms of the Creative Commons Attribution License which permits any use, distribution, and reproduction in any medium, provided the original author(s) and source are credited.

\section{References}

[1] E. Hecht, Optics, 4th Edition, Boston: Addison-Wesley, 2002.

[2] C. G. Chen, P. T. Konkola, R. K. Heilmann, C. Joo, and M. L. Schattenburg, "Nanometer accurate grating fabrication with scanning beam interference lithography," in Proc. SPIE, vol. 4936, pp. 126-134, 2002.

[3] M. Buendia, R. Cibrian, R. Salvador, C. Roldan, and J. M. Inesta, "Automatic analysis of speckle photography fringes," Applied Optics, 1997, 36(11): 2395-2400.

[4] L. He, "Vibration-compensated interferometry system using phase-modulating interference fringe subdivision technology," Applied Optics, 2006, 45(31): 7987-7992.

[5] B. Hussain, M. Ahmed, G. Hussain, M. Saleem, and M. Nawaz, "Analog processing based vibration measurement technique using Michelson interferometer," Photonic Sensors, 2013, 3(2): 137-143.

[6] B. Hussain, T. Muhammad, M. Rehan, H. Aman, M. Aslam, M. Ikram, et al., "Fast processing of optical fringe movement in displacement sensors without using an ADC," Photonic Sensors, 2013, 3(3): 241-245.

[7] L. I. Gurvits, R. A. Preston, and H. Hirabayashi, "Space VLBI as a tool for studying the early universe: first results and prospects," Astrophysics and Space Science, 1999, 269-270(0): 557-562.

[8] B. Hussain, M. Ahmed, M. Nawaz, M. Saleem, M. Razzaq, M. A. Zia, et al., "Simultaneous determination of thickness and refractive index based on time-of-flight measurements of terahertz pulse," Applied Optics, 2012, 51(21): 5326-5330.

[9] B. Hussain, M. Nawaz, M. Ahmed, and M. Y. A. Raja, "Measurement of thickness and refractive index using femtosecond and terahertz pulses," Laser Physics Letters, 2013, 10(5): 055301-1-055301-6.

[10] P. K. Rastogi, Digital speckle pattern interferometry and related techniques. New Jersey, U. S. A.: Wiley, 2001.

[11] M. A. Morsy, T. Yatagai, A. Hamza, M. A. Mabrouk, and T. Z. N. Sokkar, "Multiple-beam fizeau fringe-pattern analysis using fourier transform method for accurate measurement of fiber refractive index profile of polymer fiber," Applied Polymer Science, 2002, 85(3): 475-484.

[12] E. Berger, W. V. D. Linden, V. Dose, M. W. Ruprecht, and A. W. Koch, "Approach for the evaluation of speckle deformation measurements by application of the wavelet transformation," Applied Optics, 1997, 36(29): 7455-7460.

[13] O. S. Cedeno, M. Rivera, and R. L. Saenz, "Fast phase recovery from a single closed-fringe pattern," Journal of the Optical Society of America A, 2008, 25(6): 1361-1370.

[14] X. Yu, Y. Yao, W. Shi, Y. Sun, and D. Chen, "Study on an automatic processing technique of the circle interference fringe for fine interferometry," Optik-Internal Journal for Light and Electron Optics, 2010, 121(9): 826-830.

[15] J. Novak, "Techniques for automatic identification and numbering of interference fringes using Matlab,” http://dsp.vscht.cz/konference_matlab/ matlab02/ novak2.pdf.

[16] D. Zhang, M. Ma, and D. D. Arola, "Fringe skeletonizing using an improved derivative sign binary method," Optics and Lasers in Engineering, 2002, 37(1): 51-62.

[17] K. J. Khouzani and H. S. Zadeh, "Radon transform orientation estimation for rotation invariant texture analysis," IEEE Transactions on Pattern Analysis and Machine Intelligence, 2005, 27(6): 1004-1008.

[18] C. L. L. Hendriks, M. V. Ginkel, P. W. Verbeek, and L. J. V. Vliet, "The generalized radon transform: Sampling, accuracy and memory considerations," Pattern Recognition, 2005, 38(12): 2494-2505.

[19] C. Tang, W. Lu, Y. Cai, L. Han, and G. Wang, "Nearly preprocessing-free method for skeletonization of gray-scale electronic speckle pattern interferometry fringe patterns via partial differential equations," Optics Letters, 2008, 33(2): $183-185$.

[20] R. C. Gonzalez and R. E. Woods, Digital image processing. Upper Saddle River, U. S. A.: Prentice Hall, 2008.

[21] M. A. Ahmadshahi, "Gray-level transformation to increase the density of interferometric fringes," Applied Optics, 1991, 30(17): 2382-2385.

[22] R. A. P. Herrera, M. F. Vallejo, and M. L. Amo, "Robust fiber-optic sensor networks," Photonic Sensors, 2012, 2(4): 366-380.

[23] O. Farazao, R. M. Silva, M. S. Ferreira, J. L. Santos, 
and A. B. L. Ribeiro, "Suspended-core fibers for sensing applications," Photonic Sensors, 2012, 2(2): 118-126.

[24] B. Hussain, M. Ikram, and A. Mehmood, "A precise fast high voltage pulse measurement optical system using Kerr cell containing nitrobenzene as an optically active material," International Journal of Computer and Electrical Engineering, 2013, 5(5): 460-463.
[25] B. Hussain, M. Ahmed, M. Nawaz, and F. Gul, "Self-focusing in transformer oil with external electric field," Laser Physics, 2012, 22(12): 1815-1818.

[26] R. M. Andre, S. O. Silva, M. Becker, K. Schuster, M. Rothardt, H. Bartelt, et al., "Strain sensitivity enhancement in suspended core fiber tapers," Photonic Sensors, 2013, 3(2): 118-123. 